\title{
Phase Diagram of the Low-Density Two-Dimensional Homogeneous Electron Gas
}

\author{
N. D. Drummond and R. J. Needs \\ TCM Group, Cavendish Laboratory, University of Cambridge, J. J. Thomson Avenue, Cambridge CB3 OHE, United Kingdom
} (Received 6 February 2008; published 24 March 2009)

\begin{abstract}
We use quantum Monte Carlo methods to calculate the zero-temperature phase diagram of the twodimensional homogeneous electron gas. We find a transition from a paramagnetic fluid to an antiferromagnetic triangular Wigner crystal at density parameter $r_{s}=31(1)$ a.u. and a transition to a ferromagnetic crystal at $r_{s}=38(5)$ a.u. The fully spin-polarized fluid is never stable. We search for, but do not find, the ferromagnetic "hybrid" phase proposed by H. Falakshahi and X. Waintal [Phys. Rev. Lett. 94, 046801 (2005)].
\end{abstract}

PACS numbers: 71.10.Ca, 71.10.Pm

The two-dimensional (2D) homogeneous electron gas (HEG) is one of the most important model systems in condensed-matter physics. It is our basic model of the conduction electrons in layered semiconductor devices, and consists of a set of electrons moving in $2 \mathrm{D}$ in a uniform, inert, neutralizing background. At high densities the HEG exists in a Fermi fluid phase, but at low densities it forms a Wigner crystal to minimize the electrostatic repulsion between the electrons [1]. Classical 2D Wigner crystals can be produced in the laboratory by spraying electrons onto droplets of liquid He [2], and quantum Wigner crystals can be formed at the interface between two semiconductors [3]. As well as being objects of fundamental scientific interest, 2D Wigner crystals may be of use in quantum computing devices [4].

At high densities the fluid ground state is paramagnetic, but at low densities this phase is unstable to a ferromagnetic fluid. There is some experimental evidence that a ferromagnetic fluid is stable at densities between the paramagnetic fluid and crystal phases [5], but these data are not conclusive. Previous theoretical studies [6,7] have reported a region of stability for the ferromagnetic fluid. However, the energy differences between the various phases are small, and highly accurate calculations are required to resolve them. Another issue of recent interest is the possible existence of intermediate phases in the vicinity of the fluid-crystal transition [8-11]. In this Letter we address the issues of the stability of the ferromagnetic fluid, the fluidcrystal transition, the magnetic behavior of the crystal, and the possibility of a "hybrid" phase of the type proposed in Ref. [11].

We have performed quantum Monte Carlo (QMC) $[12,13]$ calculations for the 2D fluid and Wigner-crystal phases, achieving much smaller statistical error bars than previous studies $[6,7,14]$. We used the variational and diffusion quantum Monte Carlo (VMC and DMC) methods as implemented in the CASINO code [15]. DMC simulation is the most accurate method available for studying quantum many-body systems such as electron gases. Fermionic symmetry is imposed via the fixed-node approximation [16], in which the nodal surface is constrained to equal that of a trial wave function. The Ewald method was used to calculate the electron-electron interaction energy [17]. We used extremely flexible Slater-Jastrow-backflow [18] wave functions, and we optimized the crystal orbitals by directly minimizing the DMC energy. Our fixed-node DMC energies are therefore more accurate than those of earlier calculations. Finally, we have dealt with finite-size effects in a more sophisticated fashion than previous studies [19].

It is well established that the triangular crystal has a considerably lower energy than competing lattices in the density range of interest. A triangular lattice was therefore used in all our crystal calculations. Antiferromagnetism is frustrated on a triangular lattice, and antiferromagnetic crystals are expected to form a spin liquid [20]. Instead, we have considered antiferromagnetic crystals consisting of alternating lines of spin-up and spin-down electrons.

We used the Jastrow factor proposed in Ref. [21]. Planewave orbitals $\exp (i \mathbf{G} \cdot \mathbf{r})$ were used in the Slater wave function in our fluid calculations, while identical Gaussian orbitals $\exp \left(-C r^{2}\right)$ centered on lattice sites were used in our crystal calculations. The Slater wave function was evaluated at quasiparticle coordinates defined by the backflow (BF) transformation described in Ref. [18]. The free parameters in the Jastrow factor and $\mathrm{BF}$ function were optimized within VMC calculations by minimizing the energy [22]. The BF functions for antiparallel spins are much larger than those for parallel spins, which are already kept apart by Pauli exclusion. BF is less important in the crystal because the localization of the orbitals keeps the electrons apart. For example, at $r_{s}=$ 30 a.u. [23], BF lowers the DMC energy of the 42-electron paramagnetic fluid by $0.000036(3)$ a.u. per electron, whereas it lowers the energy of the 64-electron ferromagnetic Wigner crystal by only 0.0000023 (3) a.u. per electron. Time-step bias was removed from our final DMC energies by linear extrapolation to zero time step [24]. A target population of at least 1500 configurations was used in our production runs, making population-control bias negligible [24]. 
We optimized the Gaussian exponent $C$ in the crystal orbitals by minimizing the Slater-Jastrow DMC energy. At $r_{s}=40$ a.u., the DMC-optimized exponent of the ferromagnetic $(\mathrm{F}) \operatorname{crystal}\left(C_{\mathrm{DMC}}^{\mathrm{F}} \approx 0.0003\right.$ a.u. $)$ is smaller than the exponent obtained by minimizing the $\mathrm{VMC}$ energy $\left(C_{\mathrm{VMC}}^{\mathrm{F}} \approx 0.0006\right.$ a.u. with our Jastrow factor), which is, in turn, substantially smaller than the exponent within either Hartree or Hartree-Fock theory $\left(C_{\mathrm{H}}^{\mathrm{F}}=0.0019\right.$ a.u. $)$ [25]. The DMC energy depends much less sensitively than the VMC energy on the value of $C$ [24]. However, the energy difference between DMC results obtained with $C=$ $C_{\mathrm{DMC}}^{\mathrm{F}}$ and $C=C_{\mathrm{VMC}}^{\mathrm{F}}$ is significant, while the fixed-node error that results from using $C=C_{\mathrm{H}}^{\mathrm{F}}$ is very substantial. We find that the DMC-optimized exponents of 64-electron crystals at different densities are given by $C_{\mathrm{DMC}}^{\mathrm{F}}=$ $0.071 r_{s}^{-3 / 2}$ and that the DMC-optimized exponents for 16-, 64-, and 196-electron crystals at $r_{s}=40$ a.u. are very similar. We have therefore used $C=C_{\mathrm{DMC}}^{\mathrm{F}}$ at all densities and system sizes. For antiferromagnetic crystals we have used $C=C_{\mathrm{DMC}}^{\mathrm{AF}}=0.082 r_{s}^{-3 / 2}$.

Simulations were performed with up to $162,109,100$, and 121 electrons for the paramagnetic fluid, fully spinpolarized (ferromagnetic) fluid, antiferromagnetic crystal, and ferromagnetic crystal, respectively [24]. We eliminated single-particle finite-size effects from the fluid energies by twist averaging within the canonical ensemble [26]. Every so often during VMC or DMC simulations, an offset to the grid of $\mathbf{G}$ vectors was chosen at random in the first Brillouin zone of the simulation cell, the lowestenergy plane-wave orbitals were selected, and a short period of reequilibration was carried out. The finite-size errors in the energy per electron resulting from the compression of the exchange-correlation hole into the simulation cell and the neglect of long-ranged correlations in the kinetic energy fall off as $N^{-5 / 4}$ [19]. We therefore extrapolated our fluid energies to infinite system size by fitting our data at each density to $E_{N}=E_{\infty}-c / N^{5 / 4}$, where $E_{N}$ is the energy per electron of the $N$-electron system and $c$ and $E_{\infty}$ are fitting parameters [24]. This differs from the form of bias that has been incorrectly assumed in previous studies of the 2D HEG $[6,7,14]$.

Our DMC results for the different phases are given in Table I. Our Wigner-crystal energy data were fitted to the first five terms in the low-density expansion of the crystal energy $\quad\left(E=c_{1} r_{s}^{-1}+c_{3 / 2} r_{s}^{-3 / 2}+c_{2} r_{s}^{-2}+c_{5 / 2} r_{s}^{-5 / 2}+\right.$ $c_{3} r_{s}^{-3}$ ) [27]. The first term is the Madelung energy of the static lattice, while the second is the quasiharmonic zeropoint phonon energy. The corresponding coefficients can be determined analytically: $c_{1}=-1.106103$ and $c_{3 / 2}=$ 0.814 [28]. The remaining three coefficients were determined by fitting to our QMC data, giving $c_{2}=0.113743$, $c_{5 / 2}=-1.184994$, and $c_{3}=3.097610$ for the ferromagnetic crystal and $c_{2}=0.2662977, c_{5 / 2}=-2.63286$, and $c_{3}=6.246358$ for the antiferromagnetic crystal. We fitted
TABLE I. DMC energy as a function of $r_{s}$ for ferromagnetic and antiferromagnetic triangular Wigner crystals, and paramagnetic and fully ferromagnetic Fermi fluids. All results have been extrapolated to zero time step and infinite system size.

\begin{tabular}{lcccc}
\hline \hline \multicolumn{5}{c}{ DMC energy $\left(10^{-2}\right.$ a.u./elec.) } \\
$r_{s}$ & Ferro. crystal & Antif. crys. & Para. fluid & Ferro. fluid \\
\hline 15 & $-5.9665(1)$ & $\ldots$ & $\ldots$ & $\ldots$ \\
20 & $-4.6195(2)$ & $-4.6229(2)$ & $-4.6305(4)$ & $-4.6213(3)$ \\
25 & $-3.7731(2)$ & $-3.7751(3)$ & $-3.7774(2)$ & $-3.7740(2)$ \\
30 & $-3.1917(2)$ & $-3.1922(2)$ & $-3.1926(1)$ & $-3.1913(1)$ \\
35 & $-2.7669(1)$ & $-2.7672(1)$ & $-2.7665(1)$ & $-2.7657(1)$ \\
40 & $-2.4432(1)$ & $-2.4431(2)$ & $-2.4416(1)$ & $-2.4416(1)$ \\
45 & $-2.1881(1)$ & $-2.1875(2)$ & $\ldots$ & $\ldots$ \\
50 & $-1.9817(2)$ & $-1.9814(2)$ & $\ldots$ & $\ldots$ \\
\hline \hline
\end{tabular}

our fluid energy data to the parameterization of the correlation energy suggested by Rapisarda and Senatore [Eq. (28) of Ref. [7]]. For the paramagnetic fluid we find $a_{0}=-0.1863052$ a.u., $a_{1}=6.821839, a_{2}=0.155226$, and $a_{3}=3.423013$, where $a_{0}, a_{1}, a_{2}$, and $a_{3}$ are the free parameters in Rapisarda and Senatore's fitting form. For our ferromagnetic data we obtain $a_{0}=-0.2909102$ a.u., $a_{1}=-0.6243836, a_{2}=1.656628$, and $a_{3}=3.791685$. We find a greater difference between the energies of the paramagnetic and ferromagnetic Fermi fluids at $r_{s}=$ 20 a.u. than Attaccalite et al. [14], resulting in somewhat smaller values of the spin susceptibility.

The ground-state energies of the different phases of the 2D HEG are plotted against $r_{s}$ in Fig. 1. Unlike previous QMC studies, our statistical error bars are sufficiently small that we can resolve the energy difference between the ferromagnetic and paramagnetic fluids. We can also identify the crystallization density with much greater precision. Previous studies found crystallization to occur at $r_{s}=37(5)$ a.u. [6] and $r_{s}=34(4)$ a.u. [7]. We find the crystallization density to be $r_{s}=31$ (1) a.u., but the transition is from the paramagnetic fluid to an antiferromagnetic crystal, not the ferromagnetic fluid to the ferromagnetic crystal as found in the previous studies. Our calculations locate the density at which the paramagnetic fluid becomes unstable to the ferromagnetic fluid at about $r_{s}=40$ a.u., but at this density the Wigner crystal is more stable than either fluid phase, so we do not find a region of stability for the ferromagnetic fluid. We have looked for a region of stability for a partially spin-polarized fluid by calculating the energy of a fluid of spin polarization $\zeta=2 / 5$ at $r_{s}=35$ a.u. The DMC energy, extrapolated to zero time step and infinite size, is $-0.027666(1)$ a.u. per electron, which is not significantly less than the energies of the paramagnetic and ferromagnetic fluids, but is significantly higher than the crystal energy (see Table I). It is therefore unlikely that a region of stability exists for a partially spin-polarized fluid. We do, however, find a transition from an antiferromagnetic 


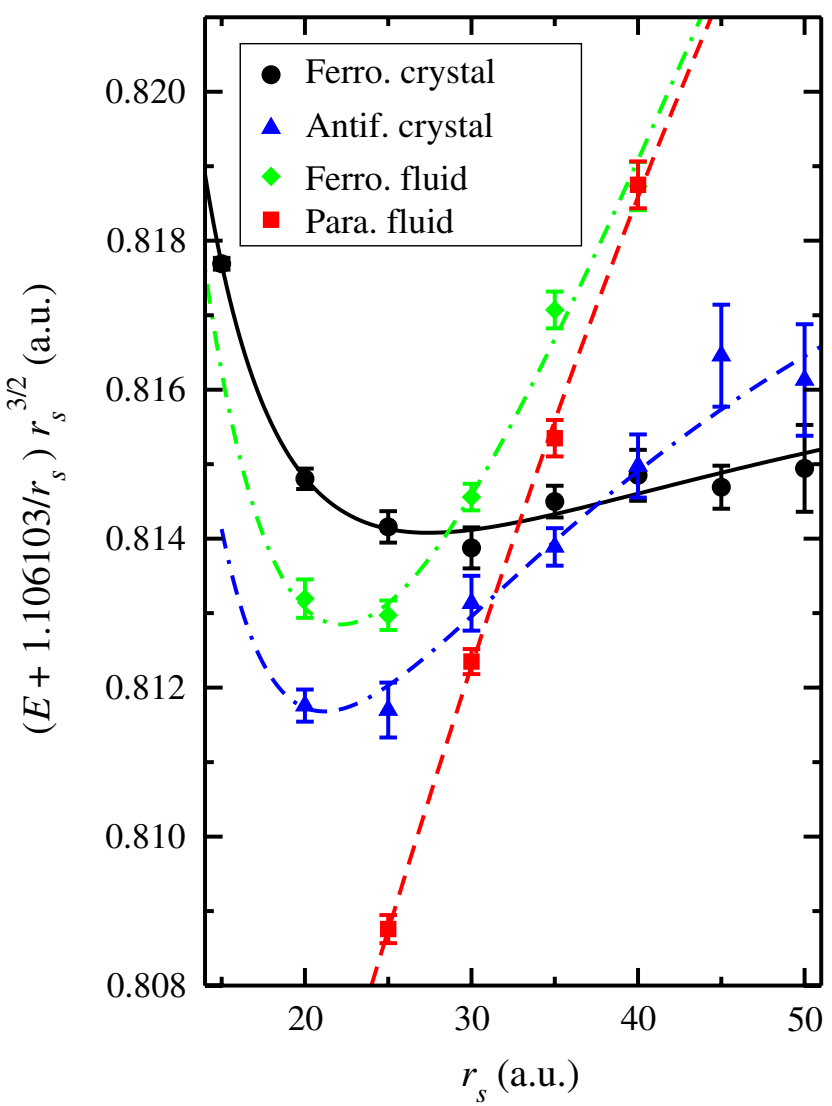

FIG. 1 (color online). DMC energy as a function of $r_{s}$ for ferromagnetic and antiferromagnetic triangular Wigner crystals and paramagnetic and fully ferromagnetic Fermi fluids. In each case the Madelung energy of a triangular crystal has been subtracted from the energy and the result has been rescaled by $r_{s}^{3 / 2}$ to highlight the differences between the curves.

Wigner crystal to a ferromagnetic one at $r_{s}=38(5)$ a.u. This is considerably higher than the density at which Bernu et al. [20] found a transition from a spin liquid to a ferromagnetic crystal using a multispin exchange model $\left(r_{s}=175\right.$ a.u. $)$. An experimental result for the crystallization density is $r_{s}=35.1(9)$ a.u. [29], which is somewhat lower than the QMC crystallization density. This suggests that the ideal 2D HEG is not a perfect model for electron layers in real semiconductor devices.

It has been argued that the transition from a 2D Fermi fluid to a Wigner crystal cannot be first order, because, at the transition density, it is energetically favorable to create boundaries between macroscopically separated regions of fluid and crystal [8]. The energy of the Bose fluid is substantially lower than that of the Fermi fluid in the vicinity of the crystallization density in $2 \mathrm{D}$, unlike $3 \mathrm{D}$ [30]. There is therefore more scope for interesting phase behavior in 2D. Various intermediate phases have been proposed in the literature, such as a hexatic phase with orientational but not translational order [9], a supersolid phase [10], or a microemulsion phase [8]. Falakshahi and
Waintal [11] have suggested that a "hybrid" phase is stable in the vicinity of the transition density from a ferromagnetic fluid to a ferromagnetic Wigner crystal. (We have found that a paramagnetic fluid is stable at this density; however, we restrict our attention to ferromagnetic HEGs to investigate the proposed hybrid phase.) The hybrid phase has the same symmetry as the Wigner crystal, but has partially delocalized orbitals.

Falakshahi and Waintal generated hybrid orbitals for the ferromagnetic $N$-electron system by solving the Schrödinger equation for a single, positively charged particle moving in a lattice of negative charges placed at the $N$ Wigner-crystal lattice sites. If the charge of the test particle is zero then the orbitals for the system are plane waves; i.e., fluid orbitals are obtained. If the charge is large then localized (crystal-like) orbitals are obtained. As the charge of the test particle is increased from zero, there must come a point at which a band gap opens up between the $N$ and $(N+1)$ th states. The set of orbitals obtained at the point at which the gap opens correspond to the hybrid phase. We have tried to find such a hybrid phase by optimizing orbitals of the form

$$
\phi(\mathbf{r})=A \exp \left(-C r^{2}\right)+\sum_{P} c_{P} \sum_{\mathbf{G} \in P} \cos (\mathbf{G} \cdot \mathbf{r})
$$

centered on the crystal lattice sites, where $P$ denotes a star of symmetry-equivalent simulation-cell $\mathbf{G}$ vectors. The coefficients $\left\{c_{P}\right\}$ and $C$ are optimizable parameters. Equation (1) is a general expansion of Wannier-like orbitals. It can therefore describe the crystal and hybrid phases, but not the fluid, since the latter corresponds to a partially filled band.

Our QMC results for ferromagnetic HEGs at $r_{s}=$ 30 a.u. obtained with different orbitals are shown in Table II. Within VMC it is possible to lower the energy significantly by optimizing the plane-wave coefficients in Eq. (1), apparently suggesting that Gaussian crystal orbitals are nonoptimal. This does not carry over to DMC, however. In fact it is more important to use Gaussian exponents optimized within DMC than it is to use either plane-wave expansions in the orbitals or BF functions, suggesting that the fixed-node errors in our crystal DMC energies are very small. We have searched for the hybrid phase by optimizing wave functions with orbitals of the form given in Eq. (1) using different starting points, but have not been able to lower the DMC energy significantly. While this does not prove the nonexistence of the hybrid phase, as searching for minima in a high-dimensional space is a very difficult problem, the fact that our extensive searches have been unable to find it makes its existence unlikely.

In summary, we have studied the zero-temperature phase behavior of the 2D HEG using QMC. We find a transition from a paramagnetic fluid to an antiferromagnetic triangular Wigner crystal at $r_{s}=31(1)$ a.u. We find no region of stability for a ferromagnetic fluid, although we find that the 
TABLE II. Non-twist-averaged QMC energies for a ferromagnetic, 121-electron HEG at $r_{s}=30$ a.u. obtained using different orbitals: crystal (Gaussian) orbitals, in which the Gaussian exponent has been optimized within VMC or DMC and hybrid (Gauss. + plane-wave) orbitals of the form given in Eq. (1) with the coefficients of 20 stars of $\mathbf{G}$ vectors optimized within VMC. The orbitals were centered on the lattice sites of a triangular crystal. VMC-optimized BF functions were used in some of the calculations. DMC energies were obtained using a time step of 1 a.u. The non-twist-averaged DMC energy of the ferromagnetic Fermi fluid at this system size and density $[-0.0319354(5)$ a.u. per electron] is relatively low due to single-particle finite-size effects, so that, if anything, finite-size effects are expected to favor the hybrid phase.

\begin{tabular}{lllc}
\hline \hline Method & \multicolumn{1}{c}{ Orbitals } & BF & Energy (a.u./elec.) \\
\hline VMC & DMC-opt. Gauss. & No & $-0.0318498(1)$ \\
VMC & DMC-opt. Gauss. + PW & No & $-0.0318499(1)$ \\
VMC & VMC-opt. Gauss. & No & $-0.0318562(1)$ \\
VMC & Gauss. + PW & No & $-0.0318619(3)$ \\
VMC & Gauss. + PW & Yes & $-0.0318713(1)$ \\
DMC & Gauss. + PW & Yes & $-0.0319180(3)$ \\
DMC & Gauss. + PW & No & $-0.0319184(3)$ \\
DMC & VMC-opt. Gauss. & No & $-0.0319184(3)$ \\
DMC & DMC-opt. Gauss. & No & $-0.0319190(2)$ \\
DMC & DMC-opt. Gauss. + PW & No & $-0.0319201(3)$ \\
\hline \hline
\end{tabular}

paramagnetic fluid is unstable to the ferromagnetic fluid at about $r_{s}=40$ a.u. and the antiferromagnetic crystal is unstable to the ferromagnetic crystal at $r_{s}=38(5)$ a.u. We find no evidence for the existence of hybrid phases of the type suggested by Falakshahi and Waintal [11], but we cannot, of course, rule out the existence of other types of intermediate phase.

We acknowledge financial support from Jesus College, Cambridge and the UK Engineering and Physical Sciences Research Council (EPSRC). Computing resources were provided by the Cambridge High Performance Computing Service and HPCx.

[1] E. P. Wigner, Phys. Rev. 46, 1002 (1934); E. P. Wigner, Trans. Faraday Soc. 34, 678 (1938).

[2] C. C. Grimes and G. Adams, Phys. Rev. Lett. 42, 795 (1979).

[3] E. Y. Andrei et al., Phys. Rev. Lett. 60, 2765 (1988); R. L. Willett et al., Phys. Rev. B 38, 7881 (1988).

[4] P. M. Platzman and M. I. Dykman, Science 284, 1967 (1999); P. Glasson et al., Phys. Rev. Lett. 87, 176802 (2001).

[5] A. Ghosh et al., Phys. Rev. Lett. 92, 116601 (2004).
[6] B. Tanatar and D. M. Ceperley, Phys. Rev. B 39, 5005 (1989).

[7] F. Rapisarda and G. Senatore, Aust. J. Phys. 49, 161 (1996).

[8] B. Spivak and S. A. Kivelson, Phys. Rev. B 70, 155114 (2004); R. Jamei, S. A. Kivelson, and B. Spivak, Phys. Rev. Lett. 94, 056805 (2005).

[9] V. Oganesyan, S. A. Kivelson, and E. Fradkin, Phys. Rev. B 64, 195109 (2001); D. G. Barci and L. E. Oxman, Phys. Rev. B 67, 205108 (2003).

[10] B. Spivak, Phys. Rev. B 67, 125205 (2003); G. Katomeris, F. Selva, and J.-L. Pichard, Eur. Phys. J. B 31, 401 (2003); Z. A. Nemeth and J.-L. Pichard, Eur. Phys. J. B 33, 87 (2003).

[11] H. Falakshahi and X. Waintal, Phys. Rev. Lett. 94, 046801 (2005); X. Waintal, Phys. Rev. B 73, 075417 (2006).

[12] W. M. C. Foulkes et al., Rev. Mod. Phys. 73, 33 (2001).

[13] D. M. Ceperley and B. J. Alder, Phys. Rev. Lett. 45, 566 (1980).

[14] C. Attaccalite et al., Phys. Rev. Lett. 88, 256601 (2002).

[15] R.J. Needs et al., CASINO version 2.1 User Manual (University of Cambridge, Cambridge, England, 2007).

[16] J. B. Anderson, J. Chem. Phys. 65, 4121 (1976).

[17] B. Wood et al., J. Phys. Condens. Matter 16, 891 (2004).

[18] P. López Ríos et al., Phys. Rev. E 74, 066701 (2006).

[19] N.D. Drummond et al., Phys. Rev. B 78, 125106 (2008).

[20] B. Bernu, L. Candido, and D. M. Ceperley, Phys. Rev. Lett. 86, 870 (2001).

[21] N. D. Drummond, M. D. Towler, and R. J. Needs, Phys. Rev. B 70, 235119 (2004).

[22] C. J. Umrigar et al., Phys. Rev. Lett. 98, 110201 (2007).

[23] Densities are given in terms of the radius $r_{s}$ of the circle that contains one electron on average. We use Hartree atomic units $\left(\hbar=|e|=m_{e}=4 \pi \epsilon_{0}=1\right)$ throughout.

[24] See EPAPS Document No. E-PRLTAO-102-073914 for auxiliary material containing detailed information about trial wave functions and time-step, population-control, and finite-size biases in the diffusion Monte Carlo energies. It also contains plots of the charge densities of crystalline phases. Finally, it contains a graph in which our energy data for the 2D homogeneous electron gas is plotted together with the results of earlier studies. For more information on EPAPS, see http://www.aip.org/pubservs/ epaps.html.

[25] J. R. Trail, M. D. Towler, and R. J. Needs, Phys. Rev. B 68, 045107 (2003).

[26] C. Lin, F. H. Zong, and D. M. Ceperley, Phys. Rev. E 64, 016702 (2001).

[27] D. Ceperley, Phys. Rev. B 18, 3126 (1978).

[28] L. Bonsall and A. A. Maradudin, Phys. Rev. B 15, 1959 (1977).

[29] J. Yoon et al., Phys. Rev. Lett. 82, 1744 (1999).

[30] S. De Palo, S. Conti, and S. Moroni, Phys. Rev. B 69, 035109 (2004). 